\title{
Las tecnologías de la información y la comunicación en el proceso docente-educativo de las ciencias sociales y empresariales como contribución a las competencias comunicativas
}

Information and communication technologies in the teaching-educational process of the social and business sciences as a contribution to communication skills

José Fabián Fonseca Vásconez. ${ }^{1}$, Hugo Stalin Yanez Rueda . ${ }^{2}$, Karol Estefanía Villacrés León. ${ }^{3}$, Andrade Alvarez Carmita Efigenia. ${ }^{4} \&$ Myriam Cecilia Sampedro Redrobán. ${ }^{5}$

Recibido: 19-03-2019 / Revisado: 27-04-209 /Aceptado: 14-05-2019/ Publicado: 05-06-2019

\section{Abstract. \\ DOI: https://doi.org/10.33262/cienciadigital.v3i2.6.563}

In a globalized world there are no borders, which means that competitiveness is more influential in the various social fields, among which education stands out as one of the pillars of development, in this text the present study tries to approach an assessment of the impact of information and communication technologies on the teaching-educational process in the careers of Social and Business Sciences as a contribution to the development of communicative competences between teachers and students. During the analysis process, the theoretical method was applied to understand facts and relevant phenomena generated by validated scientific research. Finally, the conclusions of the study are exposed, which may promote new questions susceptible to future research, considering that communicative competencies must evolve at the same pace as technological development.

1 Universidad Técnica Estatal de Quevedo, Facultad de Ciencias empresariales, Quevedo, Ecuador, jfonsecav@uteq.edu.ec

2 Universidad Tecnológica Indoamérica, Facultad de Ciencias Administrativas y Económicas, Ambato, Ecuador, hugoyanez@uti.edu.ec

3 Universidad Regional Autónoma de los Andes, Facultad de Sistemas Mercantiles, Ambato, Ecuador, karol1118_villacres@hotmail.com

4 Escuela Superior Politécnica de Chimborazo, Facultad de Administración de Empresas, Riobamba, Ecuador, carmita.alvarez@espoch.edu.ec

5 Escuela Superior Politécnica de Chimborazo, Facultad de Administración de Empresas, Riobamba, Ecuador, myriam.sampedro@espoch.edu.ec 
Keywords: Communicative Competences; Technology of the information and communication; Social and Business Sciences.

\section{Resumen.}

En un mundo globalizado no existe fronteras, lo cual incide en que la competitividad sea más influyente en los diversos ámbitos sociales entre los cuales se destaca la educación por ser considerada como uno de los pilares del desarrollo, en este texto el presente estudio intenta aproximarse a una valoración del impacto de las tecnologías de la información y la comunicación en el proceso docente-educativo en las carreras de Ciencias sociales y Empresariales como contribución al desarrollo de las competencias comunicativas entre docentes y estudiantes. Durante el proceso de análisis se aplicó el método Teórico, para comprender hechos y fenómenos relevantes generados por investigaciones científicas validadas. Finalmente, se exponen las conclusiones del estudio las mismas que pueden promover nuevas interrogantes susceptibles de investigaciones futuras, en consideración que las competencias comunicativas, deben evolucionar al mismo ritmo que el desarrollo tecnológico.

Palabras claves: Competencias Comunicativas; Tecnologías de la información y la Comunicación; Ciencias sociales y Empresariales.

\section{Introducción}

En la actualidad la humanidad enfrenta un contexto a escala global marcado por crisis económicas, sociales, políticas y ambientales, las cuales se manifiestan en las necesidades crecientes de los millones de pobres, las guerras de dominación, el cambio climático, el consumismo desmedido, así como la monopolización de la información y la cultura; lo cual conlleva a unir esfuerzos por parte de los gobiernos de los diferentes países y/o sociedades para solucionar estas problemáticas y alcanzar un desarrollo sostenible.

Esta panorámica le exige a la universidad contemporánea un proceso de contextualización en el que la accesibilidad a ella contribuya a la educación de la sociedad, para de esta forma, lograr niveles superiores de innovación, producción y divulgación de la cultura con el propósito de contribuir a salvar la humanidad.

En tal sentido, en Ecuador existe la voluntad política que se expresa en las transformaciones que ocurren en la universidad acordes con las necesidades sociales, políticas y económicas. De ahí que las ideas rectoras de la educación superior, así como el papel de los docentes, cobran un rol trascendental en el propósito de lograr un desarrollo sostenible. 
En correspondencia con esta visión, las universidades ecuatorianas direccionan sus acciones definiendo sus objetivos. Del análisis de estos se puede observar que tienen entre sus propósitos la formación óptima de los futuros egresados de las diferentes carreras. Por lo tanto, estas instituciones se manifiestan como centro de cultura social, que justamente se sostienen de los adelantos de la ciencia y la tecnología, a su vez con los vínculos que emanan entre estas y la sociedad, permitiendo el desarrollo de manera progresiva.

De tal manera, se puede afirmar que el desarrollo alcanzado por los profesionales en la universidad en su proceso de perfeccionamiento pasa indudablemente por los resultados obtenidos en durante su formación en las carreras de las Ciencias sociales y Empresariales. Es por ello que las ciencias que integran el currículo cobran una importancia relevante en el objetivo de lograr un egresado de estas carreras con la calidad necesaria para desempeñarse en su profesión.

Por esta razón, son concebidas en los planes de estudio de estas carreras, para la cual se han elaborado los programas de las asignaturas que conforman las disciplinas, manifestándose como objetivos esenciales el desarrollo de una concepción científica del mundo, así como de la personalidad, de manera integral.

Según la opinión de Diaz, A. (1997) estas razones justifican la teoría curricular empleada en la elaboración del currículo, los programas, orientaciones metodológicas, libros de textos y otros materiales de apoyo para impartir la docencia en las diversas disciplinas y además para el estudio por parte de los estudiantes, lo cual está vinculado a la necesidad de formar a futuros profesionales de esta educación en correspondencia con las condiciones históricas concretas de Ecuador y el resto del mundo.

Por tanto, el proceso de formación de los estudiantes de Ciencias Sociales y Empresariales en Ecuador se fundamenta y sostiene en los adelantos científicos y tecnológicos propios de la especialidad en cuestión y a su vez de las ciencias de la educación, en particular de la Didáctica, encargada de la dirección del proceso docente-educativo.

Se coincide con Gutiérrez (2004) al sostener que uno de los aspectos que se establecen en los planes de estudio es el empleo de las técnicas informáticas para impartir la docencia, lo cual repercute en el proceso docente-educativo, en tanto que exige la aplicación de métodos de enseñanza que favorezcan la participación activa de los estudiantes y en el que el proceso comunicativo se configure como un elemento dinamizador para el aprendizaje de los contenidos de la ciencia y la tecnología que se imparten durante la clase.

Además, este proceso formativo de los futuros egresados de la carrera de ciencias sociales y empresariales en la actualidad, está permeado del impacto de las tecnologías de la información y la comunicación como un medio para el desarrollo de las competencias 
profesionales que deben desarrollar. Según Cano (2008) una de estas son las competencias comunicativas, las cuales puede ser potenciadas mediante el uso de estos medios por parte de profesores y estudiantes.

En este texto se intenta aproximarse a una valoración del impacto de las tecnologías de la información y la comunicación en el proceso docente-educativo en las carreras de Ciencias sociales y Empresariales como contribución al desarrollo de las competencias comunicativas en los estudiantes.

\section{Metodología}

A partir de Bernal (2006), se emplean métodos teóricos como el análisis-síntesis para realizar el estudio y valoración de cómo impactan las tecnologías de la información desde la concepción del diseño de las disciplinas científicas en las carreras de Ciencias Sociales y Empresariales. El inductivo-deductivo para inferir desde procesos particulares y generalizar mediante la deducción, tal como se muestra en la siguiente gráfica.

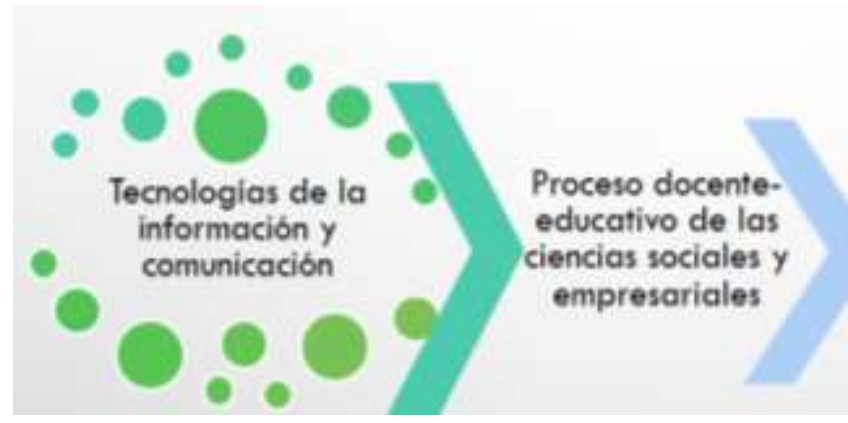

Grafica $\mathrm{N}^{\circ} 1$ Proceso metodológico
Métodos de investigación utilizados: téricos como análisis $y$ sintesis, inductivodeductivo

Elaborado por: los autores.

\section{Resultados}

En las carreras de Ciencias sociales y Empresariales que se establecen en las Universidades del Ecuador, se observan elementos comunes desde los planes de estudio que favorecen el desarrollo de las competencias comunicativas. Estos elementos se configuran mediante el impacto de las tecnologías de la información y la comunicación en este proceso formativo de los futuros egresados. Sin embargo, no todos los profesores muestran una intencionalidad didáctica y pedagógica hacia esta dirección.

Según se apunta en los planes de estudio, las disciplinas se orientan hacia la preparación de profesionales integrales comprometidos con el encargo social cuya función es la de analizar , diseñar, operar, mejorar y dirigir procesos de producción y servicios en toda la cadena de aprovisionamiento, transportación-producción-venta-servicios de posventa con el objetivo de lograr eficiencia, eficacia y competitividad mediante el análisis de relaciones que se 
presentan entre los recursos humanos, financieros, materiales, energéticos, equipamiento, información y el ambiente con un enfoque humanista, donde prevalecen criterios que sustentan los altos intereses del país.

En el micro currículo de estas carreras se señala que para esto se valen de las ciencias matemáticas, Físicas y sociales de la tecnología e informática; de conjunto con los conocimientos especializados, los principios y métodos de diseño y análisis de ingenierías, incluyendo los conocimientos necesarios en función de la sociedad ecuatoriana.

Es opinión de los autores de esta investigación que esta concepción de los futuros egresados en función de su formación, determina en su generalidad el amplio campo de acción de estos, de modo que se justifica desde el diseño curricular en el modelo del profesional el encargo social al que responden estas carreras universitarias y que para alcanzar los propósitos debe integrarse lo académico, lo investigativo y lo laboral como elementos que dinamizan y estructuran la formación de los mismos.

Por otro lado, las ciencias básicas en general deben desarrollar la base conceptual y metodológica del futuro profesional mediante la precisión del objeto de trabajo y el modo de actuación del graduado y también a la fundamentación científica de contenidos de otras disciplinas del currículo.

Además se concibe que la disciplina Informática aporta los elementos básicos para el trabajo en el campo de la computación, tales como sistemas operativos, modelación algorítmica de problemas, programación en lenguajes de alto nivel siguiendo el paradigma de la orientación a objetos y entornos de desarrollo visuales, creación de modelos de datos y construcción de las bases de datos correspondientes, así como la correcta integración de sistemas de información a otros sistemas de las organizaciones.

De conjunto con las disciplinas, se ha establecido en el plan de estudio, que la organización del proceso docente educativo debe fundamentarse en el trabajo diferenciado y personalizado con los estudiantes, determinando sus principales necesidades de formación tomando las acciones necesarias para satisfacerlos, de modo que se logre una elevada retención y permanencia de los mismos.

No obstante, estos elementos que se han referenciado, es notable como en los documentos normativos, no se explicita la necesidad de desarrollar competencias comunicativas para optimizar y perfeccionar el futuro desempeño profesional, cuestión esta que según el criterio de los autores de este texto, es imprescindible para el modo de actuación de cualquier profesional y que una de las potencialidades para alcanzar este propósito es la aplicación de las tecnologías de la información y las comunicaciones. 
Se es del criterio de que, aunque estas pueden desarrollarse mediante la ejecución de todo el currículo y desde la implementación de las estrategias curriculares, las mismas deben estar orientadas desde el plan de estudio como una de las direcciones fundamentales del modelo del profesional, de modo que se configure como una de las competencias profesionales.

Para ello se pueden utilizar las tecnologías de la información y la comunicación como medios que contribuyen al logro de estas. En este sentido existe el consenso del impacto que han tenido en cuanto a las posibilidades de transmisión de información y de apoyo para la exposición de ideas, sean estas de cualquier índole: científicas, académicas, personales, laborales. No obstante, esta visión, se considera que la comunicación no se reduce a las potencialidades de utilización de estos recursos tecnológicos, sino que ellos son medios alternativos que perfeccionan el proceso comunicativo en el contexto actual de desarrollo científico-tecnológico. Valverde (2012)

Esta idea se sostiene de la observación del proceso formativo de los futuros egresados, en el cual las tecnologías de la información y la comunicación (en lo adelante TICs) alcanzan un rol importante, en tanto que constituyen fuentes de información e interacción de los estudiantes en las actividades que desarrollan, sean estas las de estudio, consulta con profesores o compañeros de la carrera, presentación y defensa de trabajos académicos e investigativos.

Todo esto conduce al desarrollo de habilidades con el uso de las TICs y de las competencias comunicativas para alcanzar los propósitos formativos a los que aspira la sociedad en cuanto a la preparación que deben tener los profesionales. Este aspecto se ajusta al desarrollo científico-tecnológico contemporáneo a escala global, en el que las tecnologías forman parte del proceso de perfeccionamiento de la ciencia y del desarrollo social tal como lo sostiene Solomon (1995)

Entonces es innegable que el uso de las TICs no puede ser visto únicamente como fuente de información, sino como medio que contribuye de una u otra forma al desarrollo de las competencias comunicativas. Esta afirmación se puede justificar si se comprende que las TICs pueden ser el soporte para representar ideas mediante gráficos, símbolos, signos que se vinculan con la actuación comunicativa del hablante, en este caso el estudiante, y que se manifiesta en relación al texto, co-texto y al contexto en particular. Staudenmaier (1985)

Es así que un correo electrónico, una red social, una plataforma interactiva, un Applets, software, puede ser fuente de información, pero al mismo tiempo de intercambio de ideas que pueden conducir a una influencia en las esferas motivacionales y reguladoras de los individuos. En consonancia con estos elementos también se encuentra el desarrollo de la actuación comunicativa que realizan los grupos de estudiantes en la clase o en el estudio, que 
puede ser entendido como parte de lo que se llama en estos momentos trabajo colaborativo mediante el uso de las TICs.

Por tanto, el desarrollo de las competencias comunicativas se convierte en un aspecto que forma parte del actuar de los estudiantes que no puede ser dejado a un lado ni considerar su desarrollo como espontáneo. Debe constituir un objetivo del proceso formativo, no solo por las razones que asiste al pregrado de la actividad propia de los futuros egresados en este, sino por la que realizarán como profesionales una vez graduados.

De modo que más allá de las diferentes conceptualizaciones y teorizaciones en relación a las competencias comunicativas, es necesario que estas sean objetivos que se propongan los profesores a desarrollar en los estudiantes, sean estas la lingüística, la sociolingüística, la discursiva y la estratégica u otras según la visión que tenga el docente de ellas; lo importante sería al menos que los estudiantes sean capaces de alcanzar esos objetivos.

En el caso particular de las disciplinas de las ciencias básicas en las carreras referidas en este texto, los estudiantes utilizan la información existente en digital, pero intercambian sus opiniones y las defienden mediante el uso de las TICs, creando sus propias fuentes de información e intercambio, posibilitando el debate de sus ideas y reafirmando sus posiciones o transformando sus criterios en un proceso formativo que contiene en su estructura a la comunicación.

Por otro lado las diferentes plataformas de aprendizajes existentes mediante el uso de las TICs en las que se ha virtualizado una gran cantidad de conocimientos científicos y académicos que forman parte de los contenidos que se imparten en estas disciplinas, conducen al desarrollo de habilidades y a la asimilación de la materia en particular, permitiendo la interacción con otros estudiantes y profesores durante la clase, pero durante esta el docente es quien debe propiciar esta actividad de manera comunicativa y dialógica.

No hay que olvidar que justamente este proceso comunicativo y de desarrollo de las competencias comunicativas, se logra a partir de la posición que tengan los profesores del proceso formativo, los cuales dirigen el proceso, lo planifican desde la pedagogía. Si estos se sitúan como fuentes de información, de enciclopedias y los estudiantes como bancos donde se depositan los contenidos, es muy probable que estas competencias queden a lo espontáneo y no exista un desarrollo intencionado que propicie realmente el aprendizaje de las asignaturas que conforman las diferentes disciplinas y la formación integral de la personalidad de los estudiantes. Acosta, (2007)

Es conocido que muchos docentes, conciben las tecnologías de la información y la comunicación solo desde saberes prácticos, aplicados a los medios, es decir, que estudiar el desarrollo de los medios posibilita saber hacer, lo cual no refleja las relaciones sociales que 
se desarrollan, no permitiendo adquirir el conocimiento sobre la comunicación, la cienciatecnología y el proceso formativo a partir de la reflexión, el pensamiento, la modelación y la razón, con el propósito de transformar y explicar el campo en que esta se manifiesta.

En consecuencia, se enmarcan de manera esquemática en lo mediático y los medios, sin comprender los procesos y relaciones que pueden existir entre estos y las relaciones humanas. Sin percatarse que para la dirección del proceso docente-educativo la comunicación que se manifiesta entre todos los sujetos del proceso debe propiciar la adecuada interpretación y significación de la información que posibilite un clima agradable desde las emociones, sentimientos y posibilite la regulación de la conducta de todos los participantes, lo que favorecería construir en el conocimiento desde una lógica argumentativa y dialógica.

Siendo así se considera que el proceso comunicativo en el proceso docente-educativo en las carreras de Ciencias Sociales y Empresariales, no es una transmisión de conocimientos en el que el profesor es el emisor y el alumno el receptor, sino que este a la vez es emisor del conocimiento, reconstruyéndolo, y contribuyendo al desarrollo de habilidades, su personalidad y las competencias comunicativas. Hernández (2010)

Una de las tendencias actuales en la Educación Superior es la visión de la Tecnología Educativa como el modelo más eficiente para desarrollar el proceso docente-educativo, observándose una posición conductista del proceso, sin embargo, no se puede olvidar que es la actuación de cada uno de los sujetos de manera dialógica la que propicia que la implementación de las tecnologías contribuya al logro de los objetivos instructivos y educativos que se proponen en los diferentes planes de estudio.

Al respecto Torres (2016), apuntó que el modelo que se centra en los efectos, conocido también como Tecnología Educativa, tiene en sus fundamentos al conductismo, en el que se generan hábitos a partir del estímulo-recompensa introduciendo las tecnologías aparentando una participación activa de los estudiantes, en la que la actividad de estos surge siempre a partir de efectos preconcebidos en las que la reflexión, la crítica, el razonamiento y la retroalimentación se encuentra limitadas.

Aunque resulta ventajoso la utilización de las tecnologías como medios de enseñanza en contribución al logro de estrategias educativas, en particular las computadoras, los programas informáticos, la televisión, el video, el cine, la comunicación en este modelo se comporta como persuasiva y dirigista, en la que el profesor continúa teniendo el rol principal y el estudiante sigue subordinado.

Esta aseveración no significa que lo instrumental no se evidencie en el proceso docenteeducativo, de hecho esta se manifiesta en las tecnologías de la información y la comunicación, lo cual presenta un potencial educativo e instructivo importante, aportando a través de los recursos materiales el desarrollo tecnológico y a su vez científico, no solo como 
ciencia aplicada, sino como ciencia y tecnología como procesos sociales, correspondiéndose con una formación integral, en correspondencia con el encargo social asignado a la educación.

Un modelo educativo que propicia el desarrollo de una comunicación efectiva es el que se centra en los procesos, porque toma en cuenta la actitud del profesor desde que planifica su actividad docente, de modo que este se considera esencialmente comunicativo. Aquí la comunicación es democrática, se centra en la participación activa y dialógica donde los estudiantes pueden debatir y reflexionar, de modo que ocurre un intercambio entre profesoralumno, alumno-alumno y profesor-grupo de forma bidireccional, se fomenta la creatividad, así como la construcción del conocimiento y una verdadera asimilación de los contenidos, considera las experiencias, vivencias e intereses de los estudiantes, abandona el autoritarismo y el paternalismo. Es decir, se sustenta en la relación sujeto-sujeto, asumiendo la necesidad de crear significados y sentidos.

Este modelo constituye un referente fundamental en el proceso docente-educativo, en tanto que considera el diálogo, la comunicación desde la planificación de las clases, además prevé la participación activa de los estudiantes, asume lo individual y lo grupal en el aprendizaje en correspondencia con la práctica social, de modo que la socialización se configura como un componente importante y necesario para alcanzar los objetivos de la educación.

Asumir esta posición sería una vía, para desde lo teórico, concebir aspectos metodológicos necesarios para desarrollar un proceso docente-educativo que se sustente en la implementación de las tecnologías de la información y las comunicaciones como herramientas útiles para los procesos formativos de los futuros egresados de las carreras de las Ciencias Sociales y Empresariales y que supere los insuficientes resultados de la aplicación de estas en América latina.

Esta afirmación relacionada con los insuficientes resultados de la aplicación de las TICs, se puede corroborar en varias publicaciones científicas que muestran las limitaciones de considerar a las TICs o la Tecnología Educativa como modelo que solucionaría los problemas de formación de los estudiantes en un proceso docente-educativo, al respecto (Piñas Morales, Ávalos Pérez, \& Ávalos Pérez, 2017) señalan "La experiencia de incorporación de tecnologías en los sistemas educativos de América Latina y el Caribe, en los últimos veinte años ha mostrado poco efecto en la calidad de la educación".

Por tanto, se considera que estas valoraciones conforman parte de un marco teórico que los profesores de la educación Superior ecuatoriana y en particular de las carreras de Ciencias Sociales y Empresariales, pudieran emplear para realizar un análisis metodológico que se encamine al diseño y proyección de acciones orientadas hacia el cumplimiento de los objetivos de los planes de estudio de las diferentes disciplinas que conforma el currículo y de 
acuerdo a la aplicación de las TICs, pero asumiendo la necesidad, importancia y forma en que contribuirán al desarrollo de competencias comunicativas.

Para ese propósito el aspecto socio-cultural debe ser comprendido en un grado de generalidad que sea capaz al mismo tiempo de tomar en cuenta lo particular y lo singular, lo cual permita valorar el impacto del vínculo de la ciencia-tecnología, en el proceso de desarrollo de la personalidad que se manifiesta durante las actividades curriculares y extracurriculares que realizan los estudiantes orientados por los profesores. Es opinión de los autores, que estos elementos son necesarios tomarlos en cuenta en cada actividad docente como guías para valorar la manera en que influyen en los aprendizajes, en la personalidad de los estudiantes y como se enriquecen desde el intercambio entre los estudiantes y profesores.

\section{Conclusiones}

- Los profesores de las diversas disciplinas que conforman el plan de estudio de las carreras de Ciencias sociales y Empresariales tienen a su disposición las TICs como herramientas útiles para desarrollar las competencias comunicativas, siempre y cuando las actividades que se propongan a los estudiantes conduzcan al empleo de estas como apoyo al discurso de estos en las presentaciones y defensa de trabajos docentes y extradocentes.

- La integración de las TICs en las universidades, abren nuevas ventanas al mundo, que permiten a estudiantes y profesores el acceso a cualquier información necesaria en cualquier momento, la comunicación con compañeros y colegas de todo el planeta para intercambiar ideas y materiales, así como trabajar juntos.

- Considerar que las TICs son medios que favorecen la transmisión de información, pero que es el docente el encargado de posibilitar un uso de ella por parte de los estudiantes en el que se logren procesos de intercambio dialógico que contribuyan a la adecuada interpretación y significación de los conocimientos de las disciplinas científicas que estudian y favorezcan al desarrollo de competencias comunicativas.

- Es necesario que el análisis metodológico, didáctico y pedagógico que realicen los profesores de cada una de las disciplinas académicas, constituya un referente en el que se diseñen actividades docentes y extradocentes en el que los estudiantes empleen las TICs como apoyo a presentaciones y defensas de trabajos investigativos y tareas docentes, pero que la manera en que se realizan esas presentaciones mediante las TICs, sea uno de los factores que direccionen el discurso de los estudiantes en cuanto palabras que sinteticen ideas, conceptos, resultados, todo de acorde a tiempos establecidos y que favorezcan el desarrollo de competencias comunicativas. 


\section{Referencias bibliográficas.}

Acosta, H; Batista, N; (2007) La formación de valores en la Nueva Universidad: El tutor y la atención personalizada. Libro digital. Material en CD de maestría en educación superior, "La educación en valores y la tutoría en la nueva universidad cubana".

Bernal, C. (2006) Metodología de la investigación: para administración, economía, humanidades y ciencias sociales. Pearson.

Cano, G. (2008) La evaluación por competencias en la educación superior. Revista de curriculum y formación de profesorado [Consulta: 23 de marzo de 2019]. Disponible en: http://www.ugr.es/ recfpro/rev123COL1.pdf ISBN: 9789567842432

Diaz, A. (1997) Didactica y Curriculum, Editorial Paidós SAICF, 1997, [Consulta: 23 de marzo de 2019]. Impreso en México - Printed in México, ISBN: 968-853-362

Gutiérrez, R., \& Posada, R.(2004) Tendencias mundiales en educación médica. latreia [online]. 2004, vol.17, n.2 [cited 2012-11-02], pp.130-138.Available from: http://www.scielo.org.co/scielo.php?script=sci_arttext\&pid=S01 ISSN 0121-0793. Electronic Document Format (ABNT)

Hernández, J. Quiroz, R. (2010) Evaluar por competencias. [en línea] [Consulta: 22 de marzo de 2018]. Disponible en: file:// https://bit.ly/2WljocO

Piñas Morales, M., Ávalos Pérez, M., \& Ávalos Pérez, M. (2017). Saber y aprendizaje con Tecnologías de la información y la comunicación (TIC), en la Educación Superior. Revista Científica Dominio de las Ciencias, 3(2), 936-946.

Solomon, J. (1995). El estudio de la Tecnología en la educación. Alambique, 3, 13-18.

Staudenmaier, J. (1985). Technology's Storytellers. Reweaving the Human Fabric. Cambridge, MA: MIT Press.

Torres-Hernández, A. (2016). La comunicación educativa en las clases de Física con el empleo de los recursos tecnológico en la Educación Preunversitaria. Tesis en opción al grado científico de Doctor en Ciencias Pedagógicas, Universidad de Matanzas, Matanzas.

Valverde, J.(2012) Modelos de evaluación por competencias a través de un sistema de gestión de aprendizaje. [en línea] [Consulta: 22 de marzo de 2019].Disponible en: http://www.rieoei.org/rie60a03.pdf 


\section{PARA CITAR EL ARTÍCULO INDEXADO.}

Fonseca Vásconez, J. F., Yanez Rueda, H., Villacrés León, K., Carmita Efigenia, A., \& Sampedro Redrobán, M. (2019). Las tecnologías de la información y la comunicación en el proceso docenteeducativo de las ciencias sociales y empresariales como contribución a las competencias comunicativas. Ciencia Digital, 3(2.6), 262-273. https://doi.org/10.33262/cienciadigital.v3i2.6.563

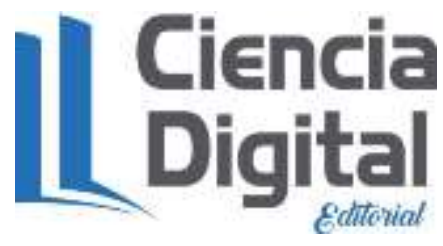

El artículo que se publica es de exclusiva responsabilidad de los autores y no necesariamente reflejan el pensamiento de la Revista Ciencia Digital.

El artículo queda en propiedad de la revista y, por tanto, su publicación parcial y/o total en otro medio tiene que ser autorizado por el director de la Revista Ciencia Digital.
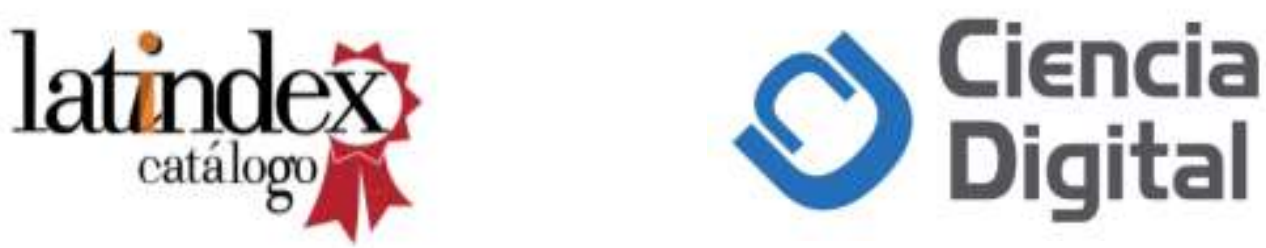